\title{
Transverse Spread and Longitudinal Dissociation in the Distal A-V Conducting System
}

\author{
Robert J. Myerburg, Kristina Nilsson, Benjamin Befeler, \\ agustin Castellanos, Jr., and Henry Gelband \\ From the Medical Service, Veterans Administration Hospital, and the Departments of \\ Medicine, Physiology, and Pediatrics, University of Miami School of Medicine, \\ Miami, Florida 33125
}

A B S T R A C T Isolated preparations of portions of the canine intraventricular conducting system were studied by microelectrode techniques in order to determine the nature of transverse spread and longitudinal dissociation of impulses in bundle branches and false tendons. Driving stimuli were delivered to an eccentric location on normal conducting tissue, and the arrival times of the propagating impulses were mapped along the length and width of the bundle branch, or along the false tendon ipsilateral and contralateral to the site of stimulation. The difference between the arrival times on the two sides was found to decrease progressively as a function of distance from the site of stimulation, the data suggesting that transverse spread of impulses involves propagation through transverse crossover points between the longitudinally oriented conducting elements. Impulses originating eccentrically became uniformly conducted across the transverse axis of bundle branches 8-15 $\mathrm{mm}$ from the level of the stimulating electrode, and of false tendons 2-4 $\mathrm{mm}$ from the stimulus site. True longitudinal dissociation, producing conduction maps different from those representing normal transverse propagation, was seen occasionally in tissue having longitudinally oriented strips of abnormal tissue. However, early premature stimulation commonly resulted in longitudinal temporal dissociation of the premature responses, possibly due to functional block in the transverse crossover fibers.

\section{INTRODUCTION}

Both the canine and human atrioventricular conducting systems are composed of bundles of conducting elements

This work was presented in part at the Annual Scientific Sessions of the American Heart Association, Anaheim, Calif., November 1971 (1971. Circulation. 44(Suppl. II) : 73.).

Received for publication 25 September 1972 and in revised form 8 December 1972. longitudinally separated from each other, at least in some portions, by connective tissue (1-3); and apparently joined periodically by transverse connections $(2,3)$. The physiological influence of this structural arrangement has been a point of speculation recently. Sherf and James (4) have considered the possibility that the subunit structure sets the stage for longitudinal dissociation of impulses introduced eccentrically into the $\mathrm{A}-\mathrm{V}$ junction or beyond, and is therefore responsible for certain forms of aberrant conduction. James and Sherf (3) more recently have further discussed the physiological implications resulting from the microanatomic features of the His bundle. In addition, an hypothesis inherently requiring longitudinal dissociation as one of its features had been offered as the explanation for reciprocal beating by earlier electrocardiographers (5). Some studies have suggested the $\mathrm{A}-\mathrm{V}$ node $(6,7)$, and perhaps the more distal conducting tissue as well (7), as the likely site(s) for the longitudinal dissociation related to reciprocal beating. However, electrophysiologists have only recently begun to study in detail the question of the possibility of longitudinal dissociation in the distal conducting system. Anderson, Greenspan, Bandura, and Fisch (8) demonstrated asynchronous conduction of premature responses in two branches of $Y$-shaped false tendon preparations, this asynchrony being a form of what we are referring to as longitudinal temporal dissociation. However, they did not observe this phenomenon in the unbranched portions of the conducting system. More recently, Spach et al. (9) provided data suggesting dissociated propagation in separate strands of conducting tissue within single false tendons of preparations composed of the left and right ventricular conducting systems and the His bundle. The present study was carried out to investigate the functional interactions between the basic subunit structure and the transverse interconnections. Anatomically less 
complex preparations than previously studied were used. This represented an attempt to delineate the electrophysiological consequences of the microanatomic structure (1-3) with a lessened probability of the presence of complexly interacting conduction pathways. Specifically, we have studied the conditions under which dissociated longitudinal conduction may occur, as well as the nature of transverse spread of impulses in single, unbranching false tendons and in bundle branches, during both regular driven stimulation and premature stimulation.

\section{METHODS}

The canine intraventricular conducting system, including bundle branches and false tendons, was used for all of these studies. The hearts were removed rapidly from adult mongrel dogs through a right thoractomy, after the animal had been anesthetized with sodium pentobarbital, $30 \mathrm{mg} / \mathrm{kg}$ i.v. The heart was placed in cool, oxygenated Tyrode's solution prepared as previously described (10), and dissection of the conducting system carried out as quickly as possible. Tissue from $48 \mathrm{dogs}$, ranging in weight from 6 $\mathrm{kg}$ to $25 \mathrm{~kg}$, was used.

The conducting system preparations were placed in a tissue bath having a $20 \mathrm{ml}$ effective volume. The bath was perfused with Tyrode's solution, flowing at a rate of 8 $\mathrm{ml} / \mathrm{min}$ at a bath temperature $36^{\circ}-37^{\circ} \mathrm{C}$. The bottom of the tissue chamber was wax-lined, and the tissue preparations were pinned in place with small steel wire pins.

Transmembrane action potentials were recorded through machine-pulled glass microelectrodes filled with $3 \mathrm{M} \mathrm{KCl}$, and having resistances between 15 and $30 \mathrm{M} \Omega$. The microelectrodes were connected to the inputs of amplifiers, having $100 \mathrm{M} \Omega$ input impedences and input capacity neutralization (Bioelectric Instruments Div., Farmingdale, N. Y.; Model $\mathrm{NF}-1$ ), by means of $\mathrm{Ag}-\mathrm{AgCl}$ junctions. The outputs of these amplifiers were connected to a group of parallel oscilloscopes for monitoring (Tektronix, Inc., Beaverton, Ore.; Model 531) and for measuring and recording transients (Tektronix, Models 564, 565, and 5030). Parameters such as the upstroke velocity $(\mathrm{dV} / \mathrm{dt})$ of phase 0 of the action potentials, conduction times, and resting and take-off potentials, used to verify the physiological state of the tissue as well as the collection of pertinent data, were measured during the course of experiments by previously described methods (11-13). Surface electrograms were recorded using close bipolar silver surface electrodes ( $0.01 \mathrm{in}$. diameter), and a high gain amplifier (Tektronix 3A3). The frequency range of the transmembrane recording system was DC to $30 \mathrm{kHz}$, and the measured rise time of a $100 \mathrm{mV}$ square wave injected into the system was $26 \mu \mathrm{s}$.

Tissue preparations. The studies were carried out on portions of the intraventricular conducting system only. The right bundle branch and the main left bundle branch were severed at the base of the muscular septum, after the right or left ventricle had been opened by techniques previously described $(10,14)$. In some studies, we used either the right bundle branch alone, or a portion of the left bundle branch and the proximal region of one of its major divisions. In other experiments, we used only a major freerunning false tendon with minimal muscle attached at both ends. A third type of preparation was composed of a bundle branch, or major division thereof, and a single peripheral ramification terminating in free wall muscle or papillary muscle. No branching false tendons were used in any of the preparations.

Stimulation of tissue. Bipolar contiguous silver surface electrodes, $0.01 \mathrm{in}$. in diameter and triple-Teflon coated except at the tips (total diameter $=0.02$ in), were used to deliver surface stimuli to the tissue. The two poles were carefully aligned parallel to the longitudinal axis of the Purkinje fiber orientation. The cathodal pole, from which measurements were made, is represented as the right pole of each pair in all illustrations. The electrodes were placed centrally or eccentrically on the segment of the conducting system studied, according to the purpose of the particular experiment.

Intracellular stimulation was also used. In these instances, after a Purkinje fiber was impaled, a relay system was used to pass a stimulating pulse through the microelectrode. The relay system permitted us to record transmembrane potentials from the same cell being stimulated except during the time that the stimulating pulse was being delivered. Pulses ranging from 1.5 to $10.0 \mathrm{~ms}$, usually less than $4 \mathrm{~ms}$, were required to depolarize cells.

During surface stimulation, it was possible to achieve local stimulation under the surface electrode by keeping the stimulus strength at 1.2 times threshold or less (see Results). Pulse duration ranged from 0.5 to $2.0 \mathrm{~ms}$, being maintained at $1.0 \mathrm{~ms}$ or less in all but six of the experiments. Threshold for premature stimulation was determined at the point on the driven transmembrane potential when complete repolarization had just occurred-that is, early phase 4. The estimation of the threshold current and multiples thereof was carried out using a variable voltage source across a fixed resistance in series with the cathodal stimulating electrode, and translating the known variables to current. A current-voltage chart was then constructed. While current output in relation to voltage was not linear throughout the entire range of voitage, it was reproducible to $\pm 0.01 \mathrm{~mA}$ at any given voltage, and therefore usable in this system. Threshold currents ranged from 0.15 to 1.10 $\mathrm{mA}$ during surface stimulation in these experiments.

Collection of data. Time and voltage transients were obtained on-line during the course of the experiments (12, 13). All permanent records were obtained photographically from oscilloscope screens. Measurements of distances on the preparations were made by means of a calibrated eyepiece in the dissecting microscope mounted over the tissue bath. The scale was divided into 10 large divisions, each of which were subdivided into 10ths. At maximum magnification, one large division was $650 \mu \mathrm{m}$, and each subdivision thereof was $65 \mu \mathrm{m}$. Time intervals between pairs of action potentials were measured from the point of maximum upstroke velocity of one to the corresponding point on the other.

Terminology. The term nonuniform wave front is used when the leading edge of the propagating wave front was not in a plane perpendicular to the long axis of the conducting fibers while the term uniform wave front is used to indicate that the leading edge was perpendicular to the long axis of the conducting fibers. The term longitudinal dissociation is used to indicate any form of asynchronous or nonuniform propagation along the longitudinal axis of a portion of the A-V conducting system. Functional block during premature propagation refers to failure of conduction across a segment of normal tissue because of refractoriness resulting from a preceding driven impulse. Transverse spread is used as a substitute for the terms transverse propagation or conduction in those setting in which we are 
unable to determine a reasonably precise pathway of conduction. It is meant to indicate empiric observations of differences in arrival times without implying anything about directional differences in true conduction velocity.

\section{RESULTS}

The basic premise determining the validity of these experiments is the assumption that local stimulation of conducting tissue is actually possible. We have tested this hypothesis with both intracellular and surface stimulation. Fig. 1 shows the results of a representative demonstration of surface stimulation at three different stimulus strengths. At 10 times threshold strength, the current density over the preparation is such that the cell impaled by the microelectrode aligned with the stimulating electrode and the cell impaled by the microelectrode on the opposite side of the false tendon are depolarized simultaneously. When the stimulus was three times threshold, there was a small interval between the ipsilateral and contralateral cell activation times. At, or just above, threshold, the interval between the activation of the ipsilateral cell and the contralateral cell was much longer. Moreover, it was the same as that obtained using intracellular stimulation of single cells in the region of the surface stimulating electrodes, while maintaining the same recording electrode relationships. Therefore, it is reasonable to assume that local stimulation can be achieved with surface electrodes as well as with intracellular stimulation. In another study, further support for this assumption was derived from the fact that we could activate a thin bundle branch without activating the subjacent muscle when using surface electrodes to deliver stimuli close to threshold strength (15).

Transierse propagation in false tendons and bundle branches. Experiments on transverse propagation during driven stimulation were carried out on 23 false tendon preparations. The basic experimental design consisted of a fixed pair of recording microelectrodes oriented transversely on the false tendons, and intracellular or surface stimulating electrodes which were moved to various sites on the false tendons (Figs. 2 and 3 ). The two recording electrodes were separated from each other by distances ranging from 0.2 to $0.8 \mathrm{~mm}$ in the 23 preparations, with a mean interelectrode distance of $0.51 \mathrm{~mm}$ and a standard deviation of $\pm 0.14 \mathrm{~mm}$. At these transverse distances between the two recording electrodes, the difference in arrival time of an impulse at the two sites of impalement was always less than $0.2 \mathrm{~ms}$ when the longitudinal distance from stimulating to recording electrodes was $4.0 \mathrm{~mm}$ or more (Fig. 2). As the longitudinal distance was decreased, and the stimulating electrode was aligned with one of the recording electrodes. the propensity for separation of arrival times increased. Thus, in 20 in the 23 experiments, separation of ar-
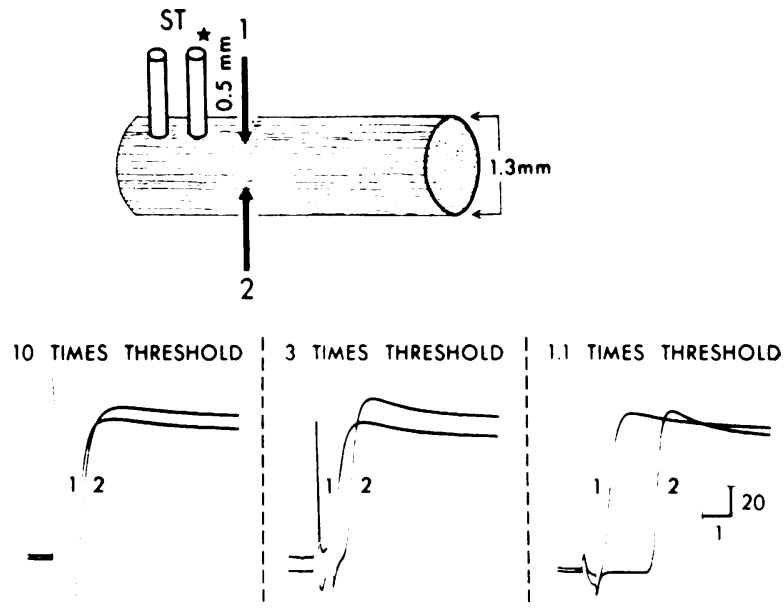

Figure 1 Local stimulation of a specific site on a portion of the conducting system. The preparation is composed of a large, free-running false tendon between the anterior division of the left bundle branch and the anterolateral papillary muscle, attached to small amounts of muscle at both ends. In this and all subsequent illustrations using this format, the drawing of the false tendon is shown as a single structural unit, but is actually composed of multiple interconnecting strands separated by connective tissue, as has been demonstrated in microanatomic studies (1-3). In the illustration, contiguous bipolar, silver surface electrodes (ST) are placed at a site on one edge of the false tendon. (N. B., the Teflon insulation on the electrodes is not represented in the illustration.) The electrodes are aligned parallel to the long axis of the fibers, and the cathodal pole is indicated by the star $(\star)$. Two recording microelectrodes are used to impale cells, one from the same side of the false tendon as ST (microelectrode no. 1) and the other opposite to it (microelectrode no. 2). Simultaneous transmembrane action potentials are recorded through microelectrodes nos. 1 and 2 at stimu'us strengths of 10 times threshold (left panel), 3 times threshold (center panel), and 1.1 times threshold (right panel). In each panel, the first of each pair of transmembrane action potentials was recorded through microelectrode no. 1 and the second through no. 2 . When the ST was replaced by a third microelectrode for intracellular stimulation, the pattern shown in the right panel was reproduced. See text for analysis of data. Horizontal calibration $=1 \mathrm{~ms}$; vertical calibration $=20 \mathrm{mV}$.

rival times at the two recording sites first exceeded 0.5 $m$ at longitudinal distances betwen stimulating and recording electrode ranging from 2.0 to $3.9 \mathrm{~mm}$ and a standard deviation of $\pm 0.42 \mathrm{~mm}$. As distances from stimulating to recording electrodes were further decreased, and when the stimulating electrodes were carefully aligned with one of the recording electrodes. disparities in arrival times at the two recording electrodes continued to increase, exceeding $1.0 \mathrm{~ms}$ at a point between 2.0 and $3.0 \mathrm{~mm}$ from stimulating to recording electrodes in 20 experiments. In 3 of the 23 experiments, separation of arrival times remained less than $0.5 \mathrm{~ms}$ at all longitudinal distances. 


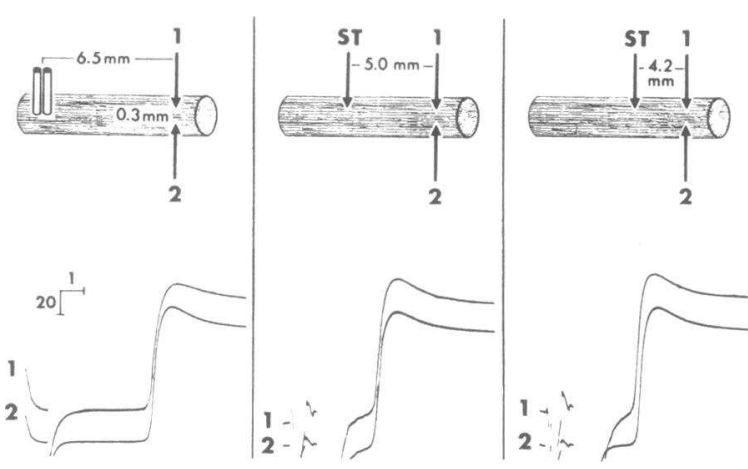

Figure 2 Surface and intracellular stimulation of a false tendon. The preparation consists of a large false tendon attached to the anteroseptal papillary muscle of the right ventricle at one end and a small segment of right ventricular free wall muscle at the other. Two microelectrodes (labeled 1 and 2) are used to record from two cells $0.3 \mathrm{~mm}$ apart. In the panel on the left, bipolar surface stimulating electrodes $6.5 \mathrm{~mm}$ from the recording microelectrodes are used to drive the preparation. In the center panel, a third microelectrode (labeled ST) is used for intracellular stimulation in line with, and $5 \mathrm{~mm}$ proximal to, microelectrode 1 . In the panel on the right, ST has been moved closer to microelectrode $1(4.2 \mathrm{~mm})$ and is still aligned with it. See text for analysis of results. Horizontal calibration $=1 \mathrm{~ms}$; vertical calibration $=20 \mathrm{mV}$.

A representative example of one of the experiments on false tendons is shown in Figs. 2 and 3. In the left panel of Fig. 2, the arrival time of the propagating impulse was identical at the sites of two microelectrodes which were $0.3 \mathrm{~mm}$ apart transversely and $6.5 \mathrm{~mm}$ distal to the surface stimulating electrodes. When intracellular stimulation was used, and the stimulating microelectrode aligned with either of the two recording microelectrodes, simultaneous arrival times at the two recording electrodes was still present when the longitudinal distance between electrodes was $5 \mathrm{~mm}$ (Fig. 2, center panel), and there was only a slight disparity of arrival times (less than $200 \mu \mathrm{s}$ ) when the distance was reduced to $4.2 \mathrm{~mm}$ (Fig. 2, right panel). However, prominent differences in arrival times occurred when the distance between the stimulating microelectrode and recording microelectrodes was less than $3 \mathrm{~mm}$. In Fig. 3, the transverse interelectrode distance for the recording electrodes was still $0.3 \mathrm{~mm}$, but the longitudinal distance between the stimulating and recording electrodes was $2.6 \mathrm{~mm}$. When the stimulating microelectrode was aligned with recording microelectrode no. 1 , activation of the cell impaled by the latter microelectrode occurred more than $1 \mathrm{~ms}$ before the contralateral cell (Fig. 3, left panel). By moving the recording microelectrode to a point on a longitudinal axis midway between the two recording electrodes (center panel), simultaneous activation of the two sites occurred. When the stimulating electrode was then aligned with the microelectrode recording from site no. 2 , that cell was activated $1 \mathrm{~ms}$ before cell no. 1 .

The transverse propagation patterns across bundle branches are different than false tendons, as might be expected by the thin, flat configurations of the bundle branch as opposed to the almost circular shape of false tendons. The differences, however, are quantitative rather than qualitative. In 15 preparations of the right bundle branch and 11 preparations of one of the divisions of the left bundle branch, mapping experiments of multiple points across the entire width and most of the length of the bundle branch were carried out. These experiments demonstrated that stimulation of an edge of a bundle branch averaging $1.2 \mathrm{~mm}$ in width (range $=$ $0.7-1.5 \mathrm{~mm}$ ) caused a nonuniform wave front to begin to propagate. As the impulse spread, the wave front became progressively more uniform across the transverse axis, requiring at least $8 \mathrm{~mm}$ to become completely uniform across the transverse axis with $15 \mathrm{~mm}$ being the maximum required in these experiments. When corrected for varying width of the bundle branches, the minimum length of bundle branch required to achieve uniform propagation across the transverse axis in these experiments calculated to be $6.4 \mathrm{~mm}$ of length per $\mathrm{mm}$ of width, with an average of $8.7 \mathrm{~mm}$ of length per $\mathrm{mm}$ of width.

The experimental technique applied to bundle branches was different than the false tendon technique for technical reasons. The inherent stability of the bundle branch on the septum allowed us to do careful and repetitive mapping studies using movable recording electrodes. The mobility of the free-running false tendons, however, caused them to be unstable enough to prevent this form of mapping, and forced us to use fixed-

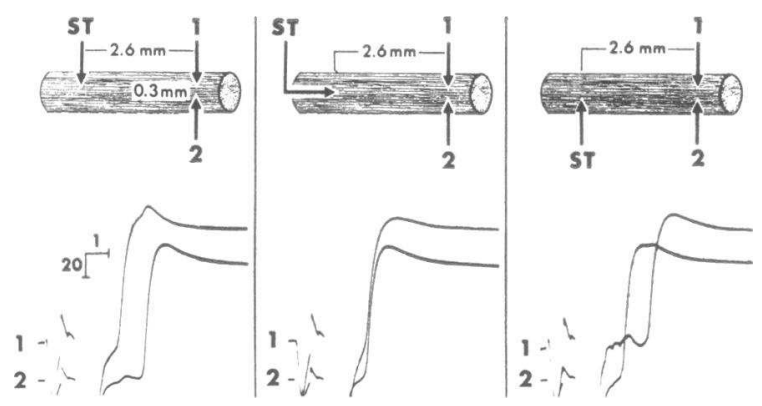

Figure 3 Intracellular stimulation at points across the transverse axis of a false tendon. The experimental preparation is the same as shown in Fig. 2. The distance from the stimulating microelectrode (ST) to the recording microelectrode is kept constant at $2.6 \mathrm{~mm}$. When ST is aligned with microelectrode 1 (left panel), activity reaches the site of microelectrode 1 first. When ST is aligned between 1 and 2 , both recording sites are activated at the same time (center panel). When ST is aligned with microelectrode 2 (right panel), activity reaches microelectrode 2 first. Horizontal calibration $=1 \mathrm{~ms}$; vertical calibration $=20 \mathrm{mV}$. See text for discussion. 
location recording electrodes and a movable stimulating electrode in order to achieve the ability to return to the same site(s).

A typical study of the distal half of the right bundle branch and its proximal free-running false tendon is demonstrated in Fig. 4. A pair of contiguous surface electrodes was used to stimulate the bundle branch proximally at a stimulus strength of 1.2 times threshold at a pulse duration of less than $1 \mathrm{~ms}$. The stimulating electrodes were located at one edge of the bundle branch. The bundle branch was stimulated at a cycle length of $1000 \mathrm{~ms}$ ( $60 \mathrm{impulses} / \mathrm{min}$ ), and two microelectrodes were used to record simultaneously from cells on both sides of the bundle branch at multiple sequential points from proximal to distal and then back again at increments of $0.2 \mathrm{~mm}$ or less. In addition, a third microelectrode was used to map arrival times across the width of the bundle branch. Only selected pairs of action potentials from the two edges of the bundle branch are shown in Fig. 4. The data shows that the difference in arrival times between the sites of the upper microelectrodes (ipsilateral to the stimulating electrodes-action potentials no. 1) and the sites of the lower microelectrodes (contralateral to the stimulating electrode-action potentials no. 2) progressively decreases from proximal to distal $-2.8 \mathrm{~ms}$ at site $\mathrm{A}, 1.8 \mathrm{~ms}$ at site $\mathrm{B}, 0.6 \mathrm{~ms}$ at site $\mathrm{C}$, and $0.3 \mathrm{~ms}$ at site $\mathrm{D}$. Thus, while longitudinal conduction progressed over almost $6 \mathrm{~mm}$ on the ipsilateral side in the first $3.8 \mathrm{~ms}$, only the first $0.7 \mathrm{~mm}$ was depolarized on the contralateral side. However, this nonuniformity of the wave of excitation became progressively less marked as a function of distance, and at about $10 \mathrm{~mm}$ distal to the site of stimulation, the wave of propagation was almost perfectly uniform across the longitudinal axis.

A serious potential pitfall in the determination of conduction velocities becomes evident in the use of experimental configurations as shown in Fig. 4. Specifically, if one calculated conduction velocity between A-1 and $\mathrm{D}-1$, the velocity would appear to be $1600 \mathrm{~mm} / \mathrm{s}$; and between the responses at A-2 and D-2, it would appear to be $3100 \mathrm{~mm} / \mathrm{s}$, with a long latency between stimulus and response at A-2. This potential hazard should be recognized in the interpretation of results obtained from this type of experimental preparation. Careful electrode

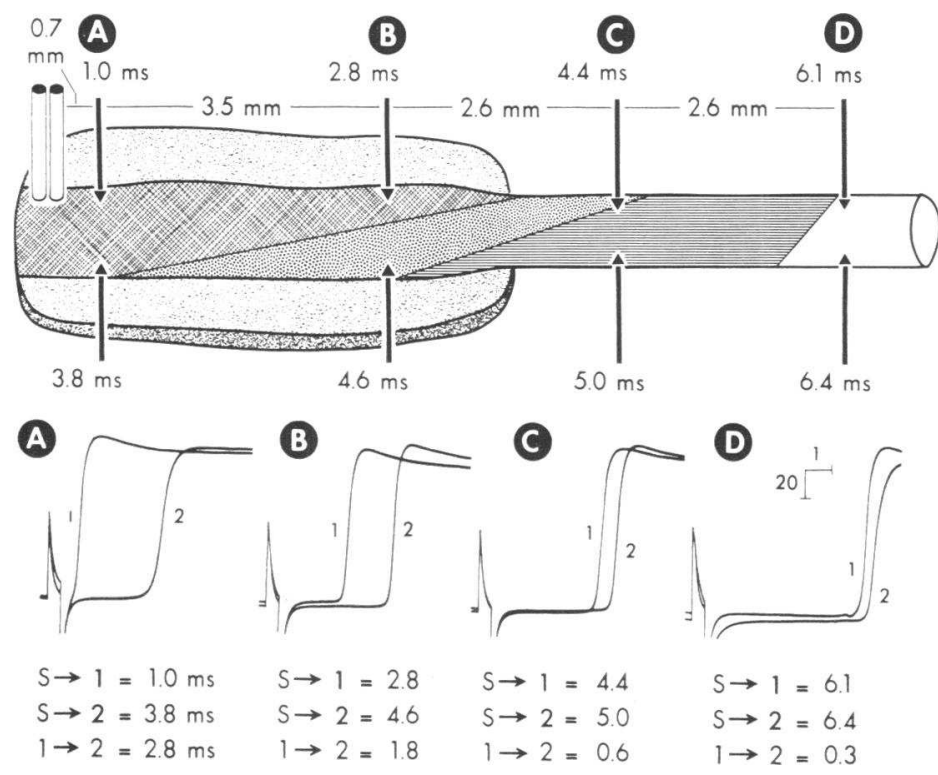

FIGURE 4 Transverse propagation across a bundle branch. The preparation consists of the distal $5 \mathrm{~mm}$ of the right bundle branch and its free running false tendon. Its termination in free wall muscle is not shown. The bundle branch is stimulated eccentrically and close to threshold by surface electrodes as shown. The time of arrival of the propagating wave front is mapped along the bundle branch and false tendon. The action potentials labeled 1 are recorded from the microelectrodes shown on the upper side of the bundle branch; those labeled 2 are recorded from the lower side. The pairs of action potentials labeled A, B, C, and D were recorded at the corresponding sites on the drawing. The oblique solid lines forming the junctions between the different types of shading on the bundle branch and false tendons are isochronic. See text for details. Horizontal calibration $=1 \mathrm{~ms}$; vertical calibration $=20 \mathrm{mV}$. $\mathrm{S} \rightarrow 1=$ time from onset of stimulus to upstroke of transmembrane potential no. $1 ; \mathrm{S} \rightarrow 2=$ time from onset of stimulus to upstroke of transmembrane potential no. 2 ; and $1 \rightarrow 2=$ time from upstroke no. 1 to upstroke no. 2. 

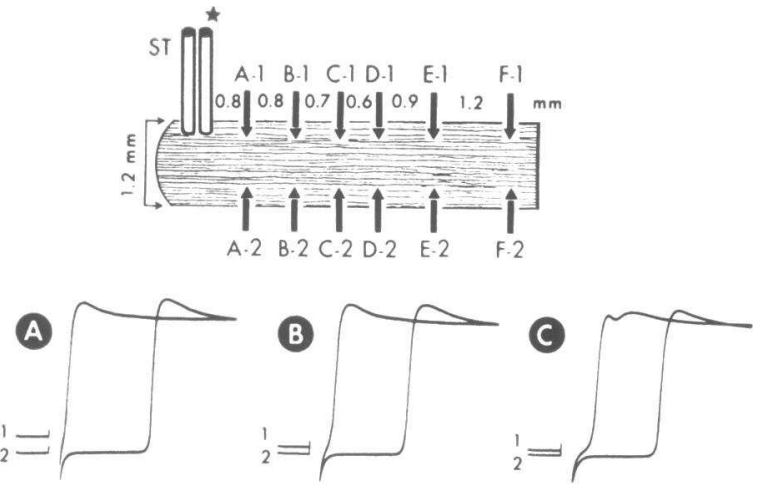

$\mathrm{S} \rightarrow 1=0.6 \mathrm{~ms}$

$\mathrm{s} \rightarrow 2=3.9 \mathrm{~ms}$

$1 \rightarrow 2=3.3 \mathrm{~ms}$

$S \rightarrow 1=0.8$

$S \rightarrow 2=37$

$1 \rightarrow 2=2.9$

$S \rightarrow 1=10$

$S \rightarrow 2=36$

$1 \rightarrow 2=26$

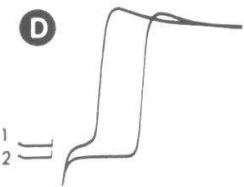

$\mathrm{S} \rightarrow 1=1.4 \mathrm{~ms}$

$\mathrm{S} \rightarrow 2=3.2 \mathrm{~ms}$

$1 \rightarrow 2=1.8 \mathrm{~ms}$
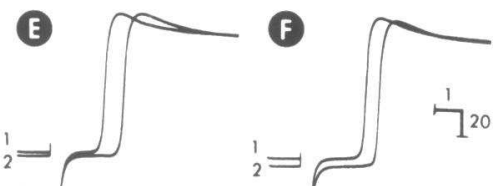

$S \rightarrow 1=1.9$

$S \rightarrow 2=2.6$

$1 \rightarrow 2=07$

$S \rightarrow 1=2.3$

$S \rightarrow 2=28$

$1 \rightarrow 2=0.5$

Figure 5 Longitudinal dissociation in an isolated bundle branch preparation. The drawing at the top of the illustration represents the proximal $6 \mathrm{~mm}$ of a right bundle branch preparation originating at the base of the muscular septum. The bundle branch was stimulated eccentrically by the surface electrodes labeled ST. Two microelectrodes are used to simultaneously record transmembrane action potentials along various sites on the two borders of the bundle branch. Microelectrode no. 1 was recording along the side ipsilateral to the stimulating electrodes, and microelectrode no. 2 was recording on the contralateral side. The transmembrane potentials shown in panels $A$ through $F$ were recorded from levels $A$ through $F$ on the preparation. Action potential no. 1 in panel A was recorded from site A-1 on the illustration, action potential no. 2 in panel A from site A-2, action potential no. 1 in panel B from site B-1, and so forth. As demonstrated by the time relationships at the various sites, the impulse traveled down the ipsilateral side of the bundle branch from the stimulating electrodes to level $\mathrm{E}$, and then turned and traveled retrograde back to level $\mathrm{A}$ on the contralateral side. See text for further description. Horizontal calibration $=1 \mathrm{~ms}$; vertical calibration $=20 \mathrm{mV}$.

alignment should be a feature of experimental designs requiring determination of conduction velocities, especially when using bundle branches and/or premature stimulation.

Longitudinal dissociation during regular rhythm (regular driven stimulation). True longitudinal dissociation, rather than nonuniform wave fronts which seem to result from certain anatomic-physiological relationships in the bundle branch (see Discussion), was recorded in five preparations of bundle branches during slow regular driven stimulation. An example is shown in Fig. 5. The preparation was a portion of a right bundle branch and the experimental design was identical to that described for the experiment shown in Fig. 4. The differences in the results, however, is striking. In the experiment shown in Fig. 5, action potentials recorded by microelectrode no. 1 (ipsilateral to the site of stimulation) showed the expected increase in propagation times from the stimulus onset to the action potential upstrokes when recording from proximal to distal. Thus, the conduction time at site $\mathrm{A}-1$ is $0.6 \mathrm{~ms}$ and at site F-1 is $2.3 \mathrm{~ms}$. However, on the contralateral side (microelectrode no. 2), the reverse occurred. The earliest site of activation on the contralateral side was at site $\mathrm{E}$, $3.8 \mathrm{~mm}$ distal to the stimulating electrodes. The activation site at site E- 2 was $2.6 \mathrm{~ms}$, and the activation times from there proximally (i.e., toward site A-2) progressively increased, reaching a maximum of $3.9 \mathrm{~ms}$ at A-2. On the portion of the bundle branch distal to level $\mathrm{E}$, the time from the stimulus to the action potential on the contralateral side (microelectrode no. 3) progressively increased as expected, and the wave front became uniform across the transverse axis of the bundle branch. Mapping of the cells in the middle of the bundle branch
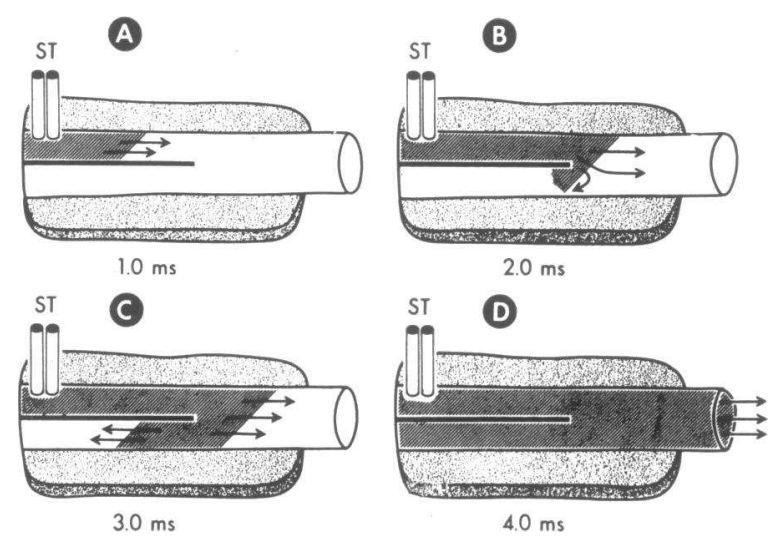

FIgURE 6 Diagrammatic representation of longitudinal dissociation in the bundle branch. The diagrams represent the preparation used for the experimental data shown in Fig. 5. The preparation consists of the right bundle branch (white) on the surface of the septal muscle (stippled). The stimulating electrodes (ST) are eccentrically located on the bundle branch, and the solid line in the center of the bundle branch represents the area of "transverse block." In panel A, $1 \mathrm{~ms}$ after stimulation, the impulse is propagating only on the side of the bundle branch ipsilateral to the stimulating electrodes. At 2 ms (panel B), the propagating impulse has just passed the abnormal area, and is beginning to invade the contralateral side. At $3 \mathrm{~ms}$ (panel C), the impulse is propagating retrogradely on the proximal contralateral side, and antegradely beyond the longitudinal block, still with a somewhat temporally dissociated wavefront across the transverse axis. After $4 \mathrm{~ms}$ (panel D), propagation is proceeding normally. It is not known whether or not such patterns may occur due to anatomic vagaries of the conducting system-see text for further comments. 
revealed a narrow longitudinal area from the most proximal portion of the bundle branch extending 3.5-4.0 mm distally in which low resting potentials could be obtained, and abortive or absent action potentials were noted. Transmembrane potentials recorded from the borders of the abnormal zone had prominent prepotentials and markedly delayed upstrokes. Similar data was obtained from the other four experiments, with variation in length of the longitudinal strip from 2.0 to 6.3 mm. Fig. 6 represents diagrammatically the nature and pathways of propagation in this form of true longitudinal dissociation. Whether or not anatomic divisions sometimes seen in the middle of bundle branches could cause this pattern in normal tissue is unknown at present. We have not observed it in this series of experiments.

Longitudinal dissociation during premature activity. Temporally dissociated longitudinal propagation could be easily induced with premature stimulation. The occurrence and degree of the dissociation was a function of the degree of prematurity. In 14 preparations (nine false tendons and five bundle branches), patterns of propagation were studied during stimulation at various degrees of prematurity. In each instance, control recordings during driven stimulation or during late premature stimulation (i.e., after complete cellular repolarization) showed no more than $0.2 \mathrm{~ms}$ of disparity in arrival time at two points separated transversely by $0.2-0.7 \mathrm{~mm}$ (average, $0.43 \mathrm{~mm}$ ). The distances between stimulating and recording electrodes ranged from 4.0 to $9.6 \mathrm{~mm}$ (average, $7.4 \mathrm{~mm}$ ). As premature stimuli were delivered progressively earlier, relative to the preceding driven response, and the premature response became weaker, the disparities in arrival times increased. The maximum temporal disparity prior to failure of conduction of the premature impulse ranged from 2.1 to $6.8 \mathrm{~ms}$ in the 14 preparations. The average maximum disparity just prior to conduction failure was $2.9 \mathrm{~ms}$.

The data shown in Fig. 7 was obtained from a representative experiment in which the stimulating electrodes were in the center of a false tendon and the bipolar surface recording electrodes $8 \mathrm{~mm}$ distally but aligned with the stimulating electrodes. Stimulus strength just in excess of threshold was used. Transmembrane action potentials were recorded at five points perpendicular to the longitudinal axis of the false tendon, and the sites recorded were less than $0.2 \mathrm{~mm}$ apart, therefore covering less than $1 \mathrm{~mm}$ of the transverse diameter of the false tendon. In the illustration, only the premature responses are shown. At a coupling interval of $300 \mathrm{~ms}$ between the driven and premature stimuli (panel A), the five premature action potentials are almost simultaneous and their upstrokes time well within the area of the surface electrogram. As the coupling intervals are progressively decreased in panels $B, C$, and $D$,

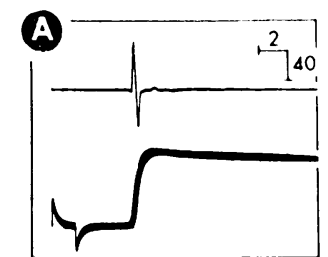

$S_{1}-S_{2}=300$

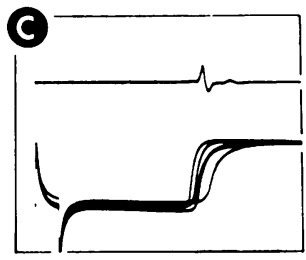

$S_{1}-S_{2}=256$

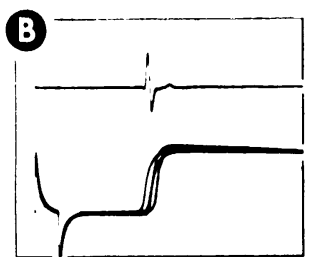

$S_{1}-S_{2}=270$

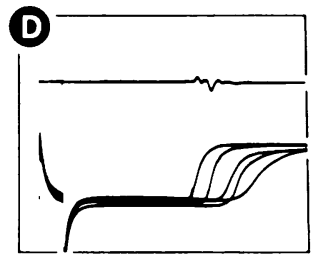

$S_{1}-S_{2}=246$
Figure 7 Dissociated propagation of premature impulses. One set of surface electrodes were used to stimulate a false tendon; and another set, $8 \mathrm{~mm}$ distally; to record the surface electrogram in the upper half of each panel. Microelectrodes were used to record transmembrane action potentials from five points within a $1 \mathrm{~mm}$ transverse line just distal to the downstream pole of the recording electrodes. The preparation was driven at a rate of $60 / \mathrm{min}$ and premature impulses applied at various coupling intervals. Only the premature responses are shown in each panel. There is progressive dissociation of action potentials across the transverse axis with increasing prematurity. Note the progressive decrease in the extracellular recording voltage and the increase in its duration with progressive prematurity of stimulation, as we have previously discussed in some detail (20). See text for further details. Horizontal calibration $=2 \mathrm{~ms}$; vertical calibration $=40 \mathrm{mV}$.

the temporal dissociation progressively increases, spanning about $4 \mathrm{~ms}$ at a coupling interval of $246 \mathrm{~ms}$ in panel D.

Another form of dissociation occasionally seen is shown in Fig. 8. The false tendon preparation is as previously described, with a distance of $2.6 \mathrm{~mm}$ between the stimulating and recording electrodes and $0.4 \mathrm{~mm}$ between the two recording microelectrodes. Only the premature responses are shown. In this experiment, at a coupling interval of $380 \mathrm{~ms}$, the premature response arrived at microelectrode no. 1 before arriving at microelectrode no. 2. The time difference was $2.8 \mathrm{~ms}$. As the coupling interval was shortened, delayed conduction to site no. 1 occurred to a much greater extent than to site no. 2, and the interval between the arrival times at the two sites progressively decreased. In panel $\mathrm{D}$, at a coupling interval of $280 \mathrm{~ms}$, the arrival times are almost simultaneous. However, as the premature coupling interval is further shortened in panels $E$ and $F$, the action potential at site no. 1 is further delayed, and site no. 1 is activated after site no. 2 . We have not been able to identify critera for functional depression of the slower path- 


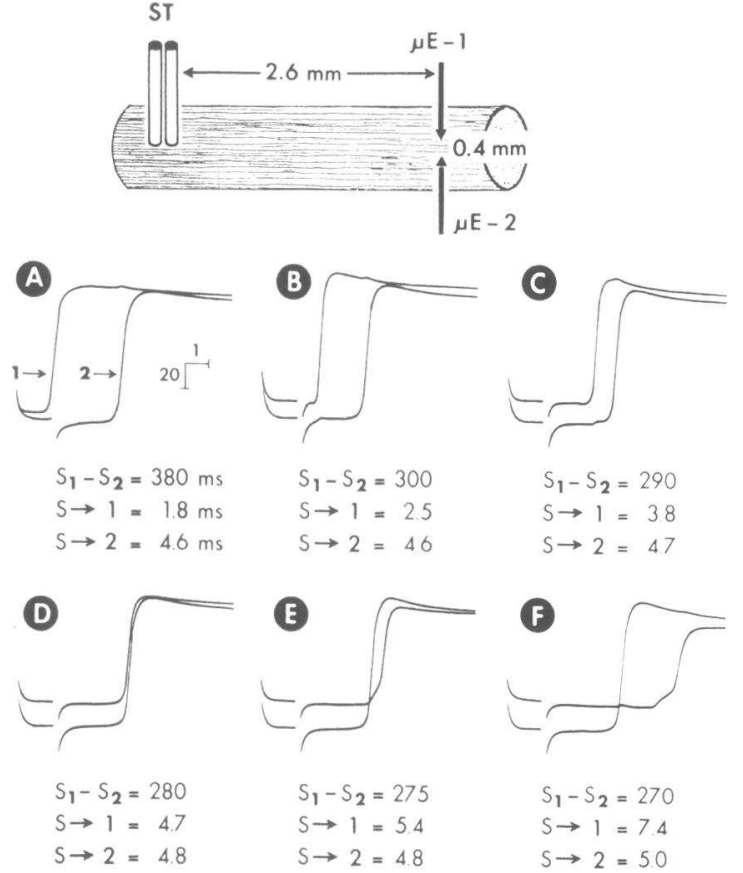

FIGURE 8 Dissociated premature propagation with reversal of conduction velocities. The stimulating electrodes are centered on a false tendon $3 \mathrm{~mm}$ from its proximal origin. They are $2.6 \mathrm{~mm}$ proximal to two microelectrodes ( $\mu \mathrm{E}-1$ and $\mu \mathrm{E}-2)$, recording from sites $0.4 \mathrm{~mm}$ apart. The false tendon was obtained from the radiation of the anterior division of the left bundle branch between the left ventricular septum and the anterolateral papillary muscle. Only the premature responses are shown. During driven stimulation at a rate of $60 / \mathrm{min}$, the two action potentials occurred less than $1 \mathrm{~ms}$ apart. At a coupling rate interval of 380 $\mathrm{ms}$ between the driven and premature stimuli, the premature response recorded by $\mu \mathrm{E}-1$ occurs $2.8 \mathrm{~ms}$ before that recorded at by $\mu \mathrm{E}-2$ (panel A). As the premature stimulus occurs progressively earlier in the cycle, delay of propagation to $\mu \mathrm{E}-1$ is much more marked than delay to $\mu \mathrm{E}-2$, and the time difference between the two responses progressively decreases, becoming $0.1 \mathrm{~ms}$ at a coupling of $280 \mathrm{~ms}$ (panel $D$ ), and then progressively increasing as further delay to $\mu \mathrm{E}-1$ occurs with further shortening of the coupling interval. The reason for the different rates of delay is unknown (see text), but the fact that it occurs indicates dissociated propagation in the system. Horizontal calibration $=1 \mathrm{~ms}$; vertical calibration $=20 \mathrm{mV}$.

way, such as differences in action potential durations, upstroke velocities, or configurations. It is, however, reminiscent of the shifting patterns of colliding impulses described by Spach et al. (9) during propagation of a premature impulse, reduced in this instance to the functionally dissociated bundles of single false tendons. Moreover, we have previously described cells close to each other in the proximal false tendons having very different action potential durations and refractory periods (10), which could acount for this behavior.

Because of the possible importance of electrotonic in- teractions in determining Purkinje fiber action potential characteristics (16), coupled with the long accepted fact that conducting tissue is at least functionally a syncytium (17), we performed experiments on preparations in which we impaled cells as close together as possible. We were attempting to enter the same subunit, or bundle of conducting fibers, within a false tendon. In three experiments, we believe we were able to enter the same subunit, with microelectrode tips approximately 60 $\mu \mathrm{m}$ apart, as confirmed by direct measurement through the calibrated eyepiece of the dissecting microscope. With proper lighting on some false tendons, under high magnification, it is possible to identify both the subunit and the dimple produced by the microelectrodes. Measuring between dimples, we were able to estimate distances with a calibrated eyepiece on the dissecting microscope. Fig. 9 demonstrates that in such an experiment, short coupling intervals could cause dissociated propagation in the parallel conducting elements within the single subunits of the false tendon. In panel $F$, at a coupling interval of $270 \mathrm{~ms}$, the dissociated arrival times within the subunit spanned $6 \mathrm{~ms}$. We were able to demonstrate
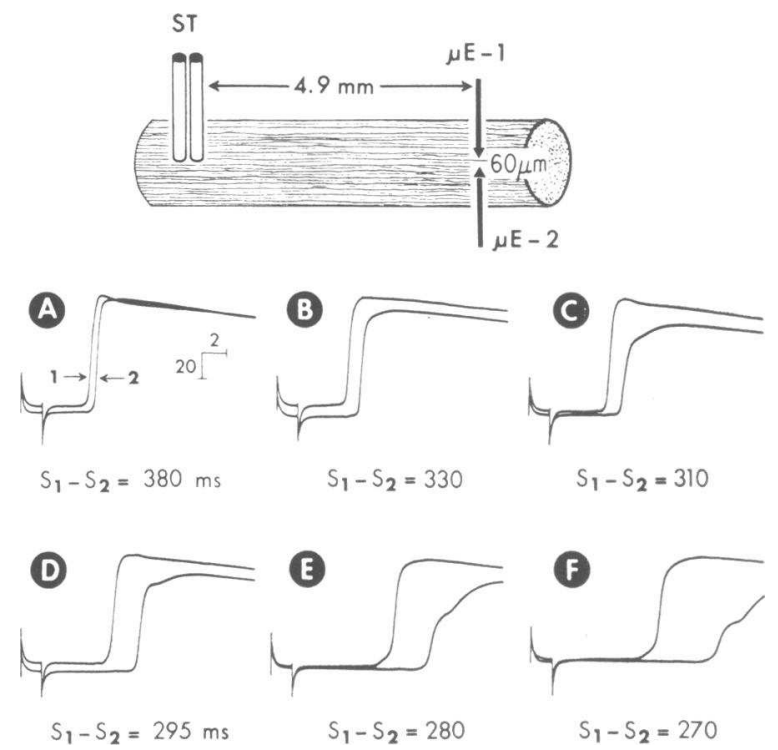

FIGURE 9 Dissociated propagation within a single subunit of a false tendon during premature activity. The basic preparation is the same as described for the experiment shown in Fig. 8, with the exception that the surface stimulating electrodes are located at the origin of the false tendon from the septum of the left ventricle, and are $4.9 \mathrm{~mm}$ from the recording electrodes. The two recording electrodes are within a single subunit of the false tendon and separated by a distance of approximately $60 \mu \mathrm{m}$. Only the premature responses are shown. Despite the fact that the microelectrodes were presumably in the same subunit, longitudinal dissociation during propagation of markedly premature impulses could still occur. See text for further details. Horizontal calibration $=2 \mathrm{~ms}$; vertical calibration $=20 \mathrm{mV}$. 
dissociation within a single subunit in two of the three experiments in which we were able to achieve the impalements. These data do not prove or disprove the possibility of transverse electrotonic interaction, but do suggest that there was no interaction between the two cells impaled during premature stimulation under the experimental conditions described.

Complete longitudinal dissociation. The data in Fig. 10 represents one of three instances of complete longitudinal dissociation observed during the course of these experiments. The preparation was composed of the anterior division of the left bundle branch and a single free-running false tendon terminating on the papillary muscle. However, while the false tendon did attach to the papillary muscle. it had no input into muscle cells (15). A very small area of septal muscle was attached to the proximal end of the preparation. Surface electrodes were used to drive the preparation at a basic cycle length of $630 \mathrm{~ms}$. and a microelectrode was used to deliver premature stimuli intracellularly. The stimulating microelectrode $(\mu \mathrm{E}-\mathrm{S})$ was aligned with recording microelectrode no. 1 at a longitudinal distance of $1.6 \mathrm{~mm}$. Recording microelectrode no. 2 was perpendicular to this line, opposite microelectrode no. 1 , at a transverse distance of $0.6 \mathrm{~mm}$. At a coupling interval of $211 \mathrm{~ms}$ (panel A), the cell at site no. 1 was depolarized by the premature impulse, but that at site no. 2 was not. At longer coupling, both cells were depolarized. At a slightly shorter coupling interval of $208 \mathrm{~ms}$ (panel B). site no. 1 was again depolarized by the premature stimulus, and no. 2 was not. However, after a small, secondary action potential (or graded response) at site no. 1. the cell at site no. 2 was depolarized. Since the muscle cells in the papillary muscle were not being depolarized even by the driven impulse, a reentrant loop involving muscle is unlikely here, because the site of the septal muscle remaining normally contains no conducting system input into muscle (15). Therefore, this appears to be an instance of complete dissociation with the conducting system, and perhaps a self-contained reentrant loop within a single branch of the conducting system.

\section{DISCUSSION}

Relevance of observations to cardiac conduction and rhythm disturbances. The results of these studies demonstrate that transverse spread of impulses arising eccentrically in bundle branches and false tendons occurs more slowly than longitudinal propagation, resulting in nonuniform wave fronts. However, the wave fronts usually become uniformly conducted after relatively short distances. While this general principle anplies both to the bundle branches and false tendons. the quantitative analysis of the data demonstrates a greater length of nonuniform conduction in the bundle branches
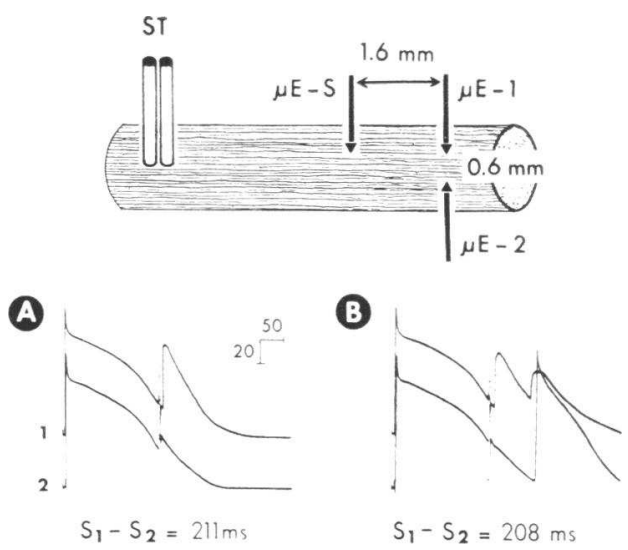

FIGURE 10 Complete longitudinal dissociation of premature impulses. In this experiment, the surface electrodes (ST) were used to deliver the driving impulse at a basic cycle length of $630 \mathrm{~ms}$. The intracellular stimulating microelectrode $(\mu \mathrm{E}-\mathrm{S})$ was used to deliver premature impulses. The distal end of the preparation did not deliver impulses into muscle to allow a muscle bridge for a reentrant loop. See text for anatomic details of the preparation. At a coupling interval of $211 \mathrm{~ms}$, the cell at $\mu \mathrm{E}-1$ responded to the premature impulse, but the cell at $\mu \mathrm{E}-2$ failed to respond (panel A). At a slightly shorter coupling interval (208 ms-panel $\mathrm{B})$, a late response at $\mu \mathrm{E}-2$ follows a secondary response at $\mu \mathrm{E}-1$ suggesting the possibility of a self-contained reentrant loop within the false tendon. Horizontal calibration $=50$ $\mathrm{ms}$; vertical calibration $=20 \mathrm{mV}$.

$(8-15 \mathrm{~mm})$ than the false tendons $(2-4 \mathrm{~mm})$. In both structures, however, true longitudinal dissociation over much longer distances (the entire length of the preparation under some conditions) occurs during the propagation of early premature impulses. as well as in damageri tissue. Longitudinal dissociation may play a role in the pathogenesis of both aberrant ventricular conduction (4) and reciprocating arrhythmias (5, 7). In the former case, our quantitative studies in regularly driven normal tissue do not support the hypothesis concerning aberrant conduction of impulses introduced eccentrically into a common pathway in the A-V junction (4) (e.g., the His bundle), but does support such an hypothesis in diseased tissue or during premature stimulation. Among possible mechanisms for reciprocating arrhythmias. moreover, prematurity-induced (Figs. 8-10) or damageinduced (Figs. 5 and 6) longitudinal dissociation could provide functionally independent parallel pathways within an anatomic unit (such as an His bundle-bundle branch-false tendon axis) or within a true common pathway such as the His bundle. Such parallel, longitudinally dissociated pathways would provide a setting for reentry, the presumed underling mechanism in reciprocity $(5,7)$. Although some data implicates pathWilys within the A-V node as potential sites of dissociation $(6,7)$, other authors suggest more distal tissue as 
dissociation within a single subunit in two of the three experiments in which we were able to achieve the impalements. These data do not prove or disprove the possibility of transverse electrotonic interaction, but do suggest that there was no interaction between the two cells impaled during premature stimulation under the experimental conditions described.

Complete longitudinal dissociation. The data in Fig. 10 represents one of three instances of complete longitudinal dissociation observed during the course of these experiments. The preparation was composed of the anterior division of the left bundle branch and a single free-running false tendon terminating on the papillary muscle. However, while the false tendon did attach to the papillary muscle, it had no input into muscle cells (15). A very small area of septal muscle was attached to the proximal end of the preparation. Surface electrodes were used to drive the preparation at a basic cycle length of $630 \mathrm{~ms}$, and a microelectrode was used to deliver premature stimuli intracellularly. The stimulating microelectrode $(\mu \mathrm{E}-\mathrm{S})$ was aligned with recording microelectrode no. 1 at a longitudinal distance of $1.6 \mathrm{~mm}$. Recording microelectrode no. 2 was perpendicular to this line, opposite microelectrode no. 1, at a transverse distance of $0.6 \mathrm{~mm}$. At a coupling interval of $211 \mathrm{~ms}$ (panel A), the cell at site no. 1 was depolarized by the premature impulse, but that at site no. 2 was not. At longer coupling, both cells were depolarized. At a slightly shorter coupling interval of $208 \mathrm{~ms}$ (panel B), site no. 1 was again depolarized by the premature stimulus, and no. 2 was not. However, after a small, secondary action potential (or graded response) at site no. 1 . the cell at site no. 2 was depolarized. Since the muscle cells in the papillary muscle were not being depolarized even by the driven impulse, a reentrant loop involving muscle is unlikely here, because the site of the septal muscle remaining normally contains no conducting system input into muscle (15). Therefore, this appears to be an instance of complete dissociation with the conducting system, and perhaps a self-contained reentrant loop within a single branch of the conducting system.

\section{DISCUSSION}

Relevance of observations to cardiac conduction and rhythm disturbances. The results of these studies demonstrate that transverse spread of impulses arising eccentrically in bundle branches and false tendons occurs more slowly than longitudinal propagation, resulting in nonuniform wave fronts. However, the wave fronts usually become uniformly conducted after relatively short distances. While this general principle applies both to the bundle branches and false tendons. the quantitative analysis of the data demonstrates a greater length of nonuniform conduction in the bundle branches
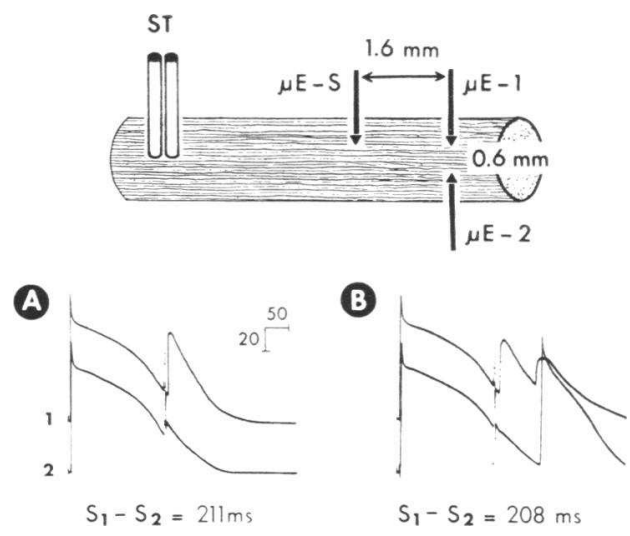

FIGURE 10 Complete longitudinal dissociation of premature impulses. In this experiment, the surface electrodes (ST) were used to deliver the driving impulse at a basic cycle length of $630 \mathrm{~ms}$. The intracellular stimulating microelectrode $(\mu \mathrm{E}-\mathrm{S})$ was used to deliver premature impulses. The distal end of the preparation did not deliver impulses into muscle to allow a muscle bridge for a reentrant loop. See text for anatomic details of the preparation. At a coupling interval of $211 \mathrm{~ms}$, the cell at $\mu \mathrm{E}-1$ responded to the premature impulse, but the cell at $\mu \mathrm{E}-2$ failed to respond (panel A). At a slightly shorter coupling interval (208 ms-panel B), a late response at $\mu \mathrm{E}-2$ follows a secondary response at $\mu \mathrm{E}-1$ suggesting the possibility of a self-contained reentrant loop within the false tendon. Horizontal calibration $=50$ $\mathrm{ms}$; vertical calibration $=20 \mathrm{mV}$.

(8-15 $\mathrm{mm}$ ) than the false tendons $(2-4 \mathrm{~mm})$. In both structures, however, true longitudinal dissociation over much longer distances (the entire length of the preparation under some conditions) occurs during the propagation of early premature impulses, as well as in damaged tissue. Longitudinal dissociation may play a role in the pathogenesis of both aberrant ventricular conduction (4) and reciprocating arrhythmias $(5,7)$. In the former case, our quantitative studies in regularly driven normal tissue do not support the hypothesis concerning aberrant conduction of impulses introduced eccentrically into a common pathway in the A-V junction (4) (e.g., the $\mathrm{His}$ bundle), but does support such an hypothesis in diseased tissue or during premature stimulation. Among possible mechanisms for reciprocating arrhythmias, moreover, prematurity-induced (Figs. 8-10) or damageinduced (Figs. 5 and 6) longitudinal dissociation could provide functionally independent parallel pathways within an anatomic unit (such as an His bundle-bundle branch-false tendon axis) or within a true common pathway such as the His bundle. Such parallel, longitudinally dissociated pathways would provide a setting for reentry, the presumed underling mechanism in reciprocity $(5,7)$. Although some data implicates pathways within the $A-V$ node as potential sites of dissociation $(6,7)$, other authors suggest more distal tissue as 
the form of true longitudinal dissociation shown in Fig. 5.

The transversely oriented crossover fibers, such as shown in Fig. 11, have a smaller cross-sectional area than the main bodies of the Purkinje fibers from which they branch. Cable theory derived for other tissue (18) predicts that cross-sectional area is inversely related to the resistance to flow of axial current. Therefore, if this same relationship applies to cardiac conducting tissue, the smaller cross-sectional area will cause the crossover fibers to have a higher resistance to axial current than the main bodies of the cells. This might become an important feature during propagation of early premature impulses in normal tissue or of driven impulses in abnormal tissue. In either instance, the accompanying depression of membrane responsiveness might create a setting in which the weakly propagating impulse could be delayed or blocked selectively at the points of higher axial resistance, thus dissociating the longitudinallyoriented elements from one another. This hypothesis could explain the mechanism of our observations on longitudinal dissociation during conduction of progressively more premature impulses, the progressive increase in the degree of temporal dissociation reflecting progressive increase in impairment of conduction across the crossover points. Ultimately, at appropriately early degrees of prematurity, the anatomic subunits within the bundle branches or false tendons (or even the individual conducting elements within a subunit) could function as independent cables within the larger structures. The foregoing hypothesis, while providing a convenient explanation for our findings, must still await experimental confirmation.

Finally, the conduction patterns that we are referring to as "longitudinal dissociation" represent a phenomenon similar to those presented by Anderson et al. in their paper on asynchronous conduction in branching false tendons (8), by Spach et al. in their studies on complexly interconnecting preparations (9), and similar observations in a study by Wennemark and Ruesta (19). Each study provides information of a different type, and a complete understanding of mechanisms and significance of longitudinal dissociation must await further study and synthesis of information.

\section{ACKNOWLEDGMENTS}

This study was supported in part by institutional research funds provided by the Miami Veterans Administration Hospital, and by Florida Heart Association Grants-in-Aid 71AG18 and 72AG25, funded by the Heart Association of Palm Beach County.

\section{REFERENCES}

1. Truex, R. C., and M. Q. Smythe. 1965. Recent observations on the human cardiac conduction system, with special considerations of the atrioventricular node and bundle. In Electrophysiology of the Heart. B. Tacardi and G. Marchett, editors. Pergamon Press Ltd., Oxford. 177.

2. Sommer, J. R., and E. A. Johnson. 1970. Comparative ultrastructure of cardiac cell membrane specializations. Am. J. Cardiol. 25: 184.

3. James, T. N., and L. Sherf. 1971. Fine structure of the His bundle. Circulation. 44: 9.

4. Sherf, L., and T. N. James. 1966. A new look at some old questions in clinical electrocardiography. Henry Ford Hosp. Med. J. 14: 265.

5. Katz, L. N., and A. Pick. 1956. Clinical Electrocardiography. Part I: The Arrhythmias. Lea \& Febiger, Philadelphia, $\mathrm{Pa} .1956$.

6. Moe, G. K., and C. Mendez. 1966. The physiologic basis of reciprocal rhythm. Prog. Cardiovasc. Dis. 8: 461.

7. Schamroth, L., and K. F. Yoshonis. 1969. Mechanisms in reciprocal rhythm. Am. J. Cardiol. 24: 224.

8. Anderson, G. J., K. Greenspan, J. P. Bandura, and C. Fisch. 1970. Asynchrony of conduction within the canine specialized Purkinje fiber system. Circ. Res. 27: 691.

9. Spach, M. S., R. C. Barr, G. S. Serwer, E. A. Johnson, and J. M. Kootsey. 1971. Collision of excitation waves in the dog Purkinje system. Circ. Res. 29: 499.

10. Myerburg, R. J., J. W. Stewart, and B. F. Hoffman. 1970. Electrophysiological properties of the canine peripheral A-V conducting system. Circ. Res. 26: 361.

11. Bigger, J. T., Jr., A. L. Bassett, and B. F. Hoffman. 1968. Electrophysiological effects of diphenylhydatoin on canine Purkinje fibers. Circ. Res. 22: 221.

12. Myerburg, R. J., J. W. Stewart, S. M. Ross, and B. F. Hoffman. 1970. On-line measurement of the duration of cardiac action potentials and refractory periods. J. Appl. Physiol. 28: 92.

13. Gelband, H., R. J. Myerburg, S. M. Ross, and B. F. Hoffman. 1970. Digital monitoring of cardiac transmembrane potential characteristics. J. Appl. Physiol. 29: 894 .

14. Myerburg, R. J., H. Gelband, and B. F. Hoffman. 1971. Functional characteristics of the gating mechanism in the canine A-V conducting system. Circ. Res. 28: 136.

15. Myerburg, R. J., K. Nilsson, and H. Gelband. 1972. The physiology of canine intraventricular conduction and endocardial excitation. Circ. Res., 30: 217.

16. Mendez, C., W. J. Mueller, J. Merideth, and G. K. Moe. 1969. Interaction of transmembrane potentials in canine Purkinje fibers and at Purkinje fiber-muscle junctions. Circ. Res. 24: 361 .

17. Hoffman, B. F., and P. F. Cranefield. 1960. Electrophysiology of the Heart. McGraw-Hill, Inc., New York.

18. Hodgkin, A. L., and W. A. H. Rushton. 1946. The electrical constants of a crustacean nerve fiber. Proc. $R$. Soc. Lond. B. 133 : 444.

19. Wennemark, J. R., and V. J. Ruesta. 1971. Microelectrode study of $2: 1$ conduction block in canine Purkinje fibers. J. Electrocardiol. 4: 50.

20. Myerburg, R. J., K. Nilsson, and R. G. Zoble. 1972. Relationship of surface electrogram recordings to activity in the underlying specialized conducting tissue. Circulation. 45 : 420. 